National Uranium Resource Evaluation

\title{
GENERAL PROCEDURE FOR CALIBRATION AND REDUCTION OF AERIAL GAMMA-RAY MEASUREMENTS: SPECIFICATION BFEC 1250-B
}

Bendix Field Engineering Corporation Grand Junction, Colorado

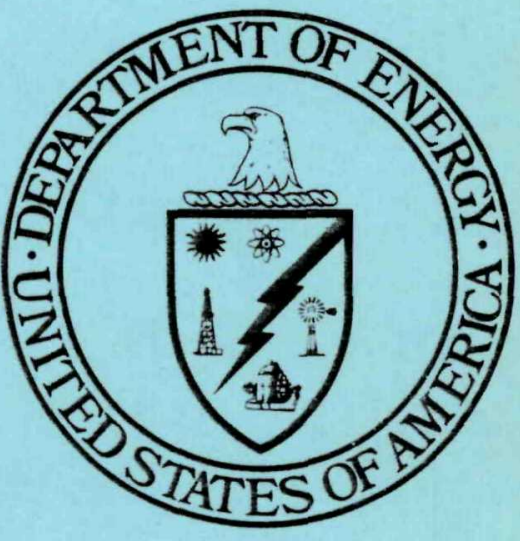

December 1983 


\section{DO NOT MICROFILM COVER}

This report was prepared as an account of work sponsored by an agency of the United States Government. Neither the United States Government nor any agency thereof, nor any of their employees, makes any warranty, express or implied, or assumes any legal liability or responsibility for the accuracy, completeness, or usefulness of any information, apparatus. product, or process disclosed in this report, or represents that its use would not infringe privately owned rights. Reference therein to any specific commercial product, process, or service by trade name, trademark, manufacturer, or otherwise, does not necessarily constitute or imply its endorsement, recommendation, or favoring by the United States Government or any agency thereof. The views and opinions of authors expressed herein do not necessarily state or reflect those of the United States Government or any agency thereof.

Available from: Technical Library

Bendix Field Engineering Corporation

P.O. Box 1569

Grand Junction, CO 81502-1569

Telephone: (303) 242-8621, Ext. 278

Price per Microfiche Copy: $\$ 4.50$ 


\section{DISCLAIMER}

This report was prepared as an account of work sponsored by an agency of the United States Government. Neither the United States Government nor any agency Thereof, nor any of their employees, makes any warranty, express or implied, or assumes any legal liability or responsibility for the accuracy, completeness, or usefulness of any information, apparatus, product, or process disclosed, or represents that its use would not infringe privately owned rights. Reference herein to any specific commercial product, process, or service by trade name, trademark, manufacturer, or otherwise does not necessarily constitute or imply its endorsement, recommendation, or favoring by the United States Government or any agency thereof. The views and opinions of authors expressed herein do not necessarily state or reflect those of the United States Government or any agency thereof. 


\section{DISCLAIMER}

Portions of this document may be illegible in electronic image products. Images are produced from the best available original document. 
David Purvance and Edward Novak

Bendix Field Engineering Corporation

Grand Junction, Colorado 81502

December 1983

Prepared for the U.S. Department of Energy

Assistant Secretary for Nuclear Energy

Grand Junction Area Office

Grand Junction, Colorado

Under Contract No. DE-AC 13 


\section{THIS PAGE WAS INTENTIONALLY LEFT BLANK}


PREFACE

The information contained in this specification was acquired over the course of the U.S. Department of Energy (DOE) National Uranium Resource Evaluation (NURE) program during the period 1974 through 1982. NURE was a program of the DOE Grand Junction Area Office to acquire and compile geologic and other information with which to assess the magnitude and distribution of uranium resources and to determine areas favorable for the occurrence of uranium in the United States. Bendix Field Engineering Corporation (BFEC) has been the operating contractor for the DOE Grand Junction facility.

The requirements stipulated herein had been incorporated as contractual specifications for the various subcontractors engaged in the aerial gamma-ray surveys, which were a major aspect of the NURE program. Although this phase of NURE activities has been completed, there exists valuable knowledge gained from these years of experience in the calibration of gamma-ray spectrometer systems and in the reduction of calibration data. Specification BFEC 1250-B is being open-filed by the U.S. Department of Energy at this time to make this knowledge available to those desiring to apply gamma-ray spectrometry to other geophysical problems.

\section{DISCLAIMER}

This report was prepared as an account of work sponsored by an agency of the United States Government. Neither the United States Government nor any agency thereof, nor any of their employees, makes any warranty, express or implied, or assumes any legal liability or responsibility for the accuracy, completeness, or usefulness of any information, apparatus, product, or process disclosed, or represents that its use would not infringe privately owned rights. Reference herein to any specific commercial product, process, or service by trade name, trademark, manufacturer, or otherwise does not necessarily constitute or imply its endorsement, recommendation, or favoring by the United States Government or any agency thereof. The views and opinions of authors expressed herein do not necessarily state or reflect those of the United States Government or any agency thereof. 


\section{THIS PAGE WAS INTENTIONALLY LEFT BLANK}




\section{CONTENTS}

Page

1.0 Introduction . . . . . . . . . . . . . . . . . . 1

2.0 Scope. . . . . . . . . . . . . . . . . . . . . . 1

3.0 Outline. . . . . . . . . . . . . . . . . . . 3

4.0 Reduction Equation ...................... . . . . 3

4.1 A Discussion of Accuracy. . . . . . . . . . . . . . 7

5.0 Error Propagation Equation . . . . . . . . . . . . . . . 8

5.1 A Discussion of Precision ................ 11

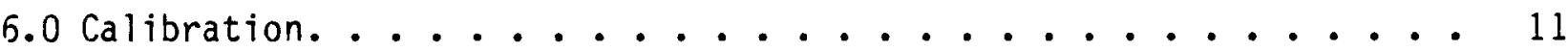

6.1 Calibration Data Acquisition. . . . . . . . . . . . . 11

6.1.1 High-Altitude Calibration. . . . . . . . . . . 11

6.1.2 Walker Field Pad Calibration . . . . . . . . 12

6.1.3 Lake Mead Dynamic Test Range Calibration . . . . . . 12

6.2 Calibration Data Reporting Requirements . . . . . . . . . 13

6.3 Calibration Data Analysis . . . . . . . . . . . . 14

6.3 .1 Notation . . . . . . . . . . . . . . . 14

6.3.2 Processing to Raw Window Count Rates . . . . . . . 15

6.3.2.1 Spectrometer Calibration Quality Control Checks . 17

6.3.3 High-Altitude Calibration Analysis . . . . . . . . 18

6.3.4 Walker Field Pad Calibration Analysis. . . . . . . 20

6.3.5 Lake Mead Dynamic Test Range Calibration Analysis. . . 25

6.3.5.1 Instrument and Cosmic Background Validation . . 26

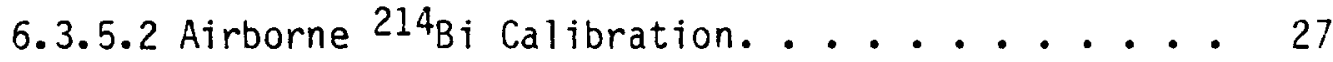

6.3.5.3 Altitude and System Normalization . . . . . 31

6.4 Statistical Adequacy Measure. . . . . . . . . . . 34

6.4 .1 Quality Flag Codes ............... 34

7.0 References..................... 36

Appendix A Fitting a Gaussian Shape to Recognizable Spectrometer Photopeaks .................. 37

Appendix B Multiple Least Squares Regression Analysis . . . . . . . . . 40

Appendix C Preparation of Average Raw Nindow Count Rates, Related Uncertainties, and Poisson Count-Rate Quality Check. . . 


\section{ILLUSTRATIONS}

Page

Figure 1. Flow Chart of Digital Processing Required by Specification BFEC 1200-C. . . . . . . . . . . 2

2. Reduction Equation ................ 5

3. Functional Form of Reduction Equation. . . . . . . 6

4. Error Propagation Equation . . . . . . . . . . . 9

5. Functional Form of Errors in Error Propagation Equation ................. 10

6. Walker Field Pad Infinite-Plane Normalization Curve

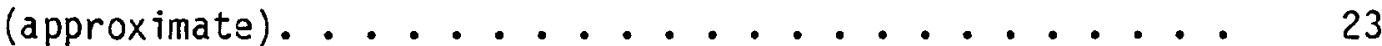

TABLES

Table 1. Energy Limits for Spectral Windows . . . . . . . . 16

2. Photopeaks for $E_{0}, \Delta E$ Definition. . . . . . . . . . 16

3. Walker Field Pad Concentrations. . . . . . . . . 21

4. Lake Mead Assigned 122-Meter Effective Ground Concentrations .................. 


\subsection{INTRODUCTION}

Several subcontractors utilizing unique hardware participated in the aerial radiometric reconnaissance surveys which were conducted in support of the National Uranium Resource Evaluation (NURE) program. To provide continuity to the program's product, it was necessary to specify a standardized calibration and processing procedure for the various systems. The purpose of this specification was to detail the calibration, calibration documentation, and data processing that were required of those subcontractors participating in the NURE aerial survey program.

The aerial radiometric reconnaissance survey program had as its purpose and end product the acquisition of knowledge of the surface distribution of naturally occurring radioactive potassium, uranium, and thorium (KUT) as a function of geographic position. To realize a credible product on such a massive scale as the NURE program, extreme attention to detail was required with respect to all aspects of calibration, data acquisition, quality control, reduction, and storage. This specification was designed to ensure attention to detail, and hence, to aid in the production of a credible block of geologic information.

Within the boundaries of a measurement's detail lie its quantitative and qualitative specifics. Quantitative analysis determines a measurement's magnitude. Qualitative analysis addresses a measurement's precision and accuracy. The scope of this specification was consciously expanded beyond quantitative analysis to include a rigorous treatment of precision and a mention of accuracy. This was done with the explicit intention of defining the inherent limitations in these measurements. Knowledge of such limitations places these measurements in their proper perspective relative to geologic interpretation.

\subsection{SCOPE}

Figure 1 is a schematic which shows the digital processing required by Specification BFEC 1200-C, General Specification for Airborne Geophysical Surveys. The enhanced boxes within this scheme are the areas addressed herein. As shown in Figure 1, the input to these procedures are raw field data. Specification BFEC 1200-C specifies the processing procedures to be followed in general terms. This specification, BFEC 1250-B, formulates the details of this processing, and the calibration and quality checks required for its realization. 


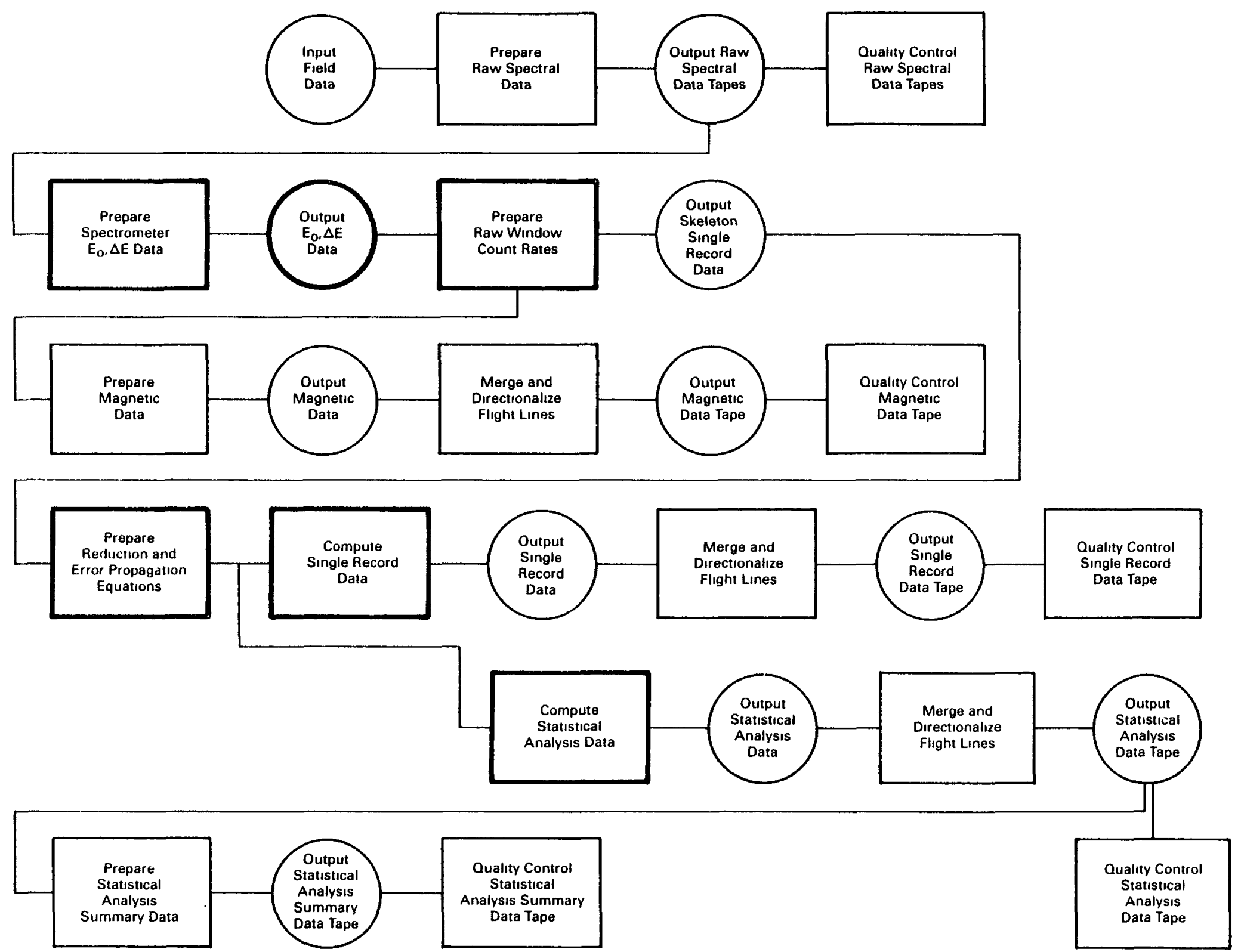

Figure 1. Flow Chart of Digital Processing Required by Specification BFEC 1200-C 


\subsection{OUTLINE}

Three interrelated processes are described. In order of presentation these processes are the following:

1. Reduction

2. Error Propagation

3. Calibration

First, the reduction equation (paragraph 4.0) is specified. This equation symbolically defines the corrections and normalizations to be applied to raw spectrometric field data. The associated error propagation equation (paragraph 5.0) is then given. This equation defines the precision, and hence the statistical adequacy, of reduced quantities. The output of these combined processes are the basic information to be posted on the single record reduced data tape referenced in paragraph 4.7, Specification BFEC 1200-C.

Having specified the reduction and error propagation equations, their application to survey measurements requires symbols to be replaced with raw survey data and numerical calibration constants. In this vein, the calibration and data analysis procedures are then described (paragraph 6.0); these descriptions constitute the bulk of this specification. Collection of calibration data and BFEC reporting requirements are detailed. Then a step-by-step analysis of these calibration data is described in the same sequence as required in the reduction and error propagation equations.

Quality control procedures are described at all appropriate points within the reduction and calibration portions of this specification. Their purpose is to provide repeated assurance of proper instrumentation calibration during a field survey and accurate system calibration.

\subsection{REDUCTION EQLATION}

Calibration and data reduction represent a blend of physical theory and experiment which precisely and accurately describe, isolate, and quantify all active parameters involved in a measurement. The product of these activities is a set of equations and constants which, when applied to the raw output from a detection device, yields the information sought. Infinitely precise and accurate equations and constants are impossible to realize, and, in practice, some portions of precision and accuracy are traded for tractability. 
Over the years, experience has led to a tractable reduction equation for aerial gamma-ray spectrometer measurements. This reduction equation is cast in the form of linear equations, and, since multielement and total-activity information are extracted from each raw spectral sample, these equations are described in matrix notation. The reduction equation that shall be applied to unit raw spectral samples is

$$
\bar{C}_{a}=N[S(\vec{R}-\bar{B})-\vec{A}]
$$

where

$$
\begin{aligned}
& \bar{C}_{a}=\text { apparent concentration vector } \\
& N=\text { altitude and system normalization matrix } \\
& S=\text { Compton and cosmic stripping matrix } \\
& \bar{R}=\text { raw window count-rate vector } \\
& \bar{B}=\text { instrument background vector } \\
& \bar{A}=\text { airborne } 214_{B} i \text { vector }
\end{aligned}
$$

Figures 2 and 3 provide the explicit functional form of equation (4.1). Figure 2 iliustrates that there are five reduction steps to be applied to raw spectral data. These are:

1. Preparation of raw window count rates.

2. Removal of instrument background.

3. Compton and cosmic stripping.

4. Removal of airborne $214_{B i}$.

5. Altitude and instrument normalization.

The first reduction step involves locating and summing channel counts for the various windows utilized to detect KUT radioelements and total activity. Also, instrument dead time is accounted for by converting observed counts to deadtime-corrected counts per second (cps). The next two reduction steps remove instrument, Compton, and cosmic backgrounds present in the various windcws. Vote that a Compton correction is not applied to the total-activity wincow, and that the Compton correction removes the scattered airborne $214_{B i}$ component fresent in $40_{K}$ and ${ }^{208} \mathrm{Tl}$ windows. The fourth reduction step removes the airborne $214_{B i}$ component present in the terrestrial $214_{B i}$ window. Reduction steps (2) through (4) are defined as background corrections in the sense 


$$
\bar{C}_{\mathrm{a}}=N[S(\bar{R}-\bar{B})-\bar{A}]
$$

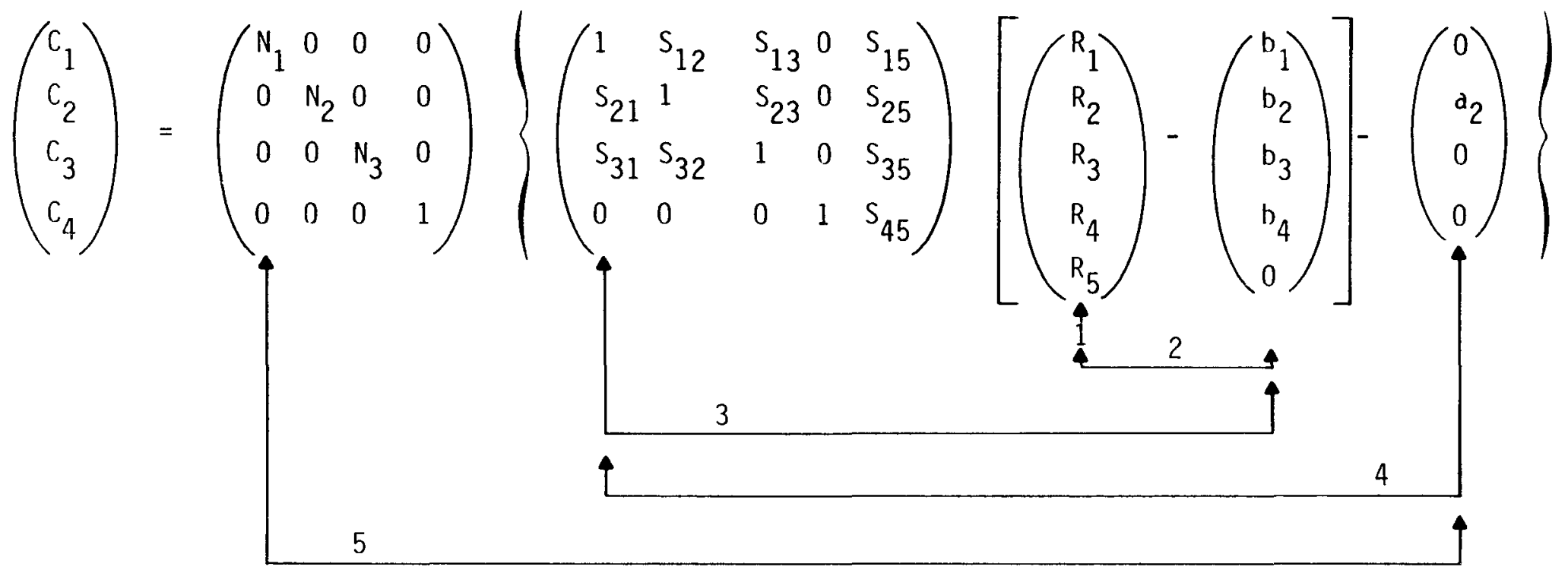

Reduction Steps

1. Preparation of raw window count rates

2. Removal of instrument background

3. Compton and cosmic stripping

4. Removal of airborne ${ }^{214} \mathrm{Bi}$

5. Altitude and instrument normalization
$\mathrm{R}_{1}=$ raw window count rate for ${ }^{40} K$

$R_{2}=$ raw window count rate for ${ }^{214} \mathrm{Bi}$

$R_{3}^{2}=$ raw window count rate for ${ }^{208} \mathrm{Tl}$

$\mathrm{R}_{4}=$ raw window count rate for total activity

$R_{5}=$ raw window count rate for cosmic activity

Figure 2. Reduction Equation 
Matrix "I (normalization corrections)

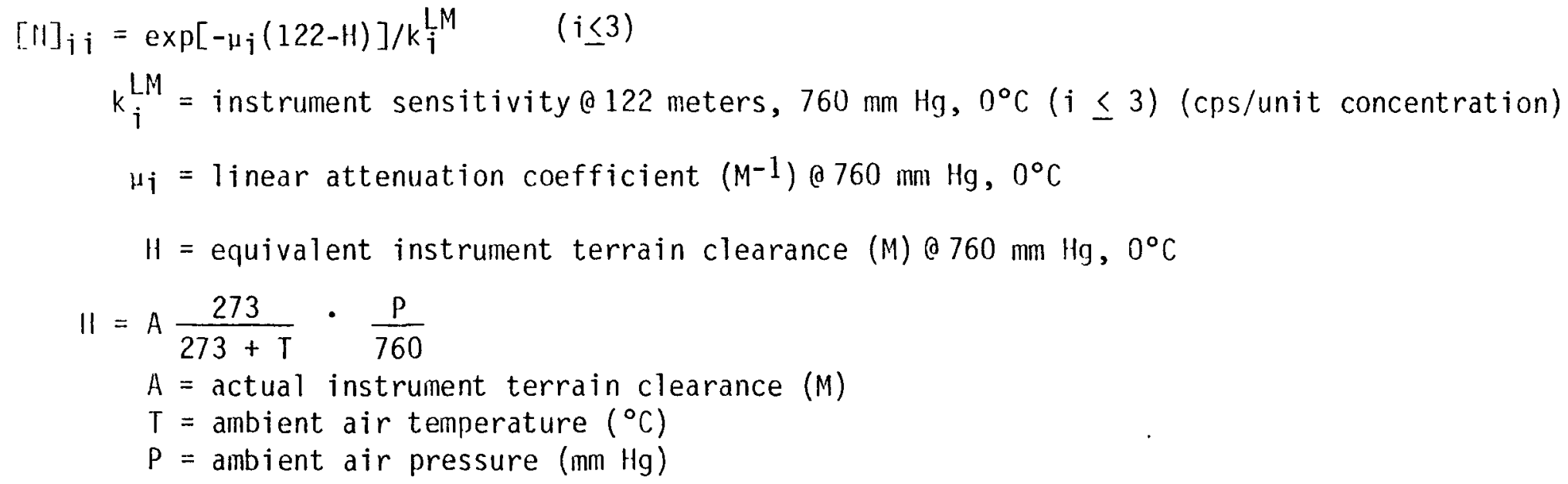

Matrix $S$ (Compton and cosmic corrections)

$S_{i j}=$ Compton stripping coefficients $(i \leq 3, j \leq 3), S_{i 5}=$ Cosmic stripping coefficients

Vector $\bar{R}$ (raw count rates)

$R_{j}=$ raw window count rates normalized to counts per second (cps)

Vector $\bar{B}$ (background correction)

$b_{i}=$ instrument background count rate $(\mathrm{cps}) \quad(i \leq 4)$

Vector $\bar{A}$ (airborne ${ }^{214_{B i} \text { correction) }}$

$$
a_{2}=\frac{R_{4}^{2 \pi}-b_{4}^{2 \pi}+S_{45}^{2 \pi} R_{5}^{2 \pi}-f(H)\left(R_{4}^{4 \pi}-b_{4}^{4 \pi}+S_{45}^{4} R_{5}^{4 \pi}\right)}{g(H)-0.19 f(H)}
$$

$f(H)=2 \pi$ effective shielding efficiency function $g(H)=2 \pi-t o-4 \pi$ effective volume coupling function 
that they remove the multiple-source, Compton, and cosmic-interference phenomena present in each window. The final reduction step is the application of two normalization corrections. The first correction analytically continues background-subtracted count rates to a constant survey datum, and the second divides out instrument sensitivity. These normalization corrections are necessary to provide amplitude continuity among the various aerial systems participating in the NURE surveys. Note that a system-sensitivity normalization for total activity is not applied.

\subsection{A DISCUSSION OF ACCURACY}

Apparent rather than true concentrations are intentionally referenced on the left-hand side of equation (4.1). This is done as a reminder that equation (4.1) contains some inaccuracies, including the following:

1. Secular equilibrium:

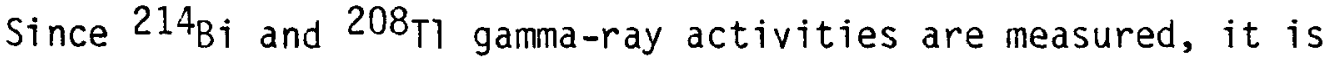
assumed that these activities are equal to those of their parent isotopes, $238 \mathrm{U}$ and $232 \mathrm{Th}$, respectively.

2. Ground-source geometry:

The source geometry assumed in equation (4.1) is a homogeneous half space.

3. Added absorbers:

Equation (4.1) assumes no additional attenuation and scattering due to added surface absorbers such as vegetative cover.

4. Soil properties:

Soil density and moisture content assumed in equation (4.1) are those present at the Lake Mead Dynamic Test Range on the day of the calibration sorties.

5. Source geometry for airborne ${ }^{214_{B i} \text { : }}$

The source distribution assumed in equation (4.1) is the linear portion of the airborne $214 \mathrm{Bi}$ distribution present at the Lake Mead Dynamic Test Range on the day of the calibration sorties. Ground-source nonuniformity, disequilibrium, terrain, vegetation, soil properties, and nonuniform airborne sources can all interplay simultaneously in a geologic environment. Isolating these effects to extract absolute groundsource concentrations using aerial gamma-ray measurements is an impossible 
task. These are the major inherent interpretational limitations involved in aerial gamma-ray spectrometer data. While such inaccuracies complicate this particular measurement's geologic interpretation, virtually every geophysical measurement suffers similar afflictions.

\subsection{ERROR PROPAGATION EQUATION}

Gamma-ray activity is a statistical phenomenon predictable only in terms of the Poisson probability density function. This phenomenon adds a unique twist to the interpretation of radiometric data as compared with more conventional geophysical measurements. To aid interpretation, a confidence computation is necessary. The error propagation equation provides such a computation from which signal-to-noise ratios (SNR) for reduced quantities are estimated.

The error propagation equation that shall be applied to each unit sample reduction is

$$
\bar{E}=M_{1}\left[M_{2}\left(\bar{V}_{1}+\bar{V}_{2}\right)+M_{3} \bar{V}_{3}+\bar{V}_{4}\right]+M_{4} \bar{V}_{5}
$$

Figures 4 and 5 delineate the explicit form for equation (5.1). Figure 4 also breaks down the error propagation equation in terms of the reduction steps as given in paragraph 4.0 .

The first precision error introduced in equation (5.1) is due to Poisson counting fluctuations in the raw window count rates. A second but less important precision error is present in reduction step (1). This is the resultant scale error introduced by the system dead-time correction. Since instrument background also fluctuates according to Poisson counting statistics, its removal in the reduction equation adds uncertainty to the results. These uncertainties add in quadrature as is indicated in error propagation step (2). Precision errors associated with Compton and cosmic stripping are formulated in error propagation step (3). These errors arise from imprecisions involved in determining Compton and cosmic stripping constants by experimental calibration, and uncertainties in instrument-background-corrected count rates. Removal of an airborne $214_{B i}$ source also adds uncertainty to terrestrial $214_{B i}$. This is accounted for in error propagation step (4). Finally, altitude and system normalization corrections add imprecision to reduced quantities. These uncertainties are accounted for in error propagation step (5). 


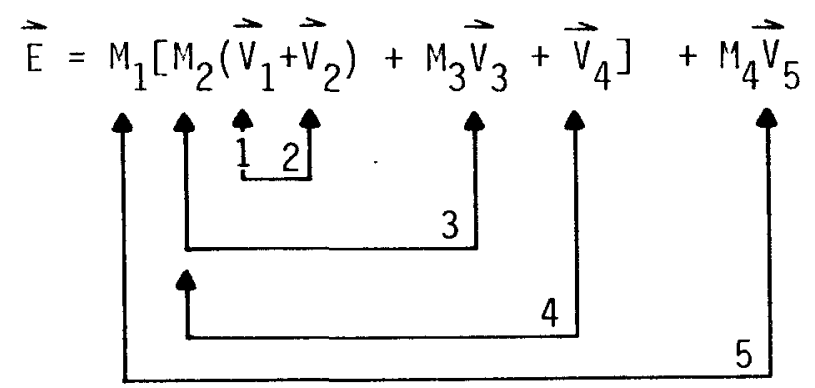

$\vec{E}=4 \times 1$ vector whose elements contain the square of the error in' the elements of $\vec{C}_{a}$ $H_{1}=4 \times 4$ matrix whose elements contain the square of the elements in $\mathrm{N}$ $H_{2}=4 \times 5$ inatrix whose elements contain the square of the elements in $S$ $I_{3}=4 \times 5$ matrix whose elements contain the square of the error in the elements of $S$ $M_{4}=4 \times 4$ matrix whose elements contain the square of the error in the elements of $N$ $\vec{V}_{1}=5 \times 1$ vector whose elements contain the square of the error in the elements of $\vec{R}$ $\vec{V}_{2}=5 \times 1$ vector whose elements contain the square of the error in the elements of $\vec{B}$ $\vec{V}_{3}=5 \times 1$ vector whose elements contain the square of the elements in $\vec{R}-\vec{B}$ $\vec{V}_{4}=4 \times 1$ vector whose elements contain the square of the error in the elements of $\vec{A}$ $\vec{V}_{5}=4 \times 1$ vector whose elements contain the square of the elements in $[S(\vec{R}-\vec{B})-\vec{A}]$

Figure 4. Error Propagation Equation 
Matrix $M_{3}$ (error in stripping matrix)

$$
\left[M_{3}\right]_{i j}=e^{2}\left(S_{i j}\right) \quad(i \leq 4, j \leq 5)
$$

Matrix $M_{4}$ (error in normalization matrix)

$$
\begin{aligned}
& {\left[M_{4}\right]_{i j}=[N]_{i j}^{2}\left[e^{2}\left(k_{i}^{L M}\right) /\left(k_{i}^{L M}\right)^{2}+(122-H)^{2} e^{2}\left(\mu_{j}\right)\right] \quad(i \leq 3)} \\
& \quad e\left(k_{i}^{L M}\right) \text { numerically computed from multiple least squares (MLS) algorithm } \\
& \quad e\left(\mu_{i}\right) \text { numerically computed from MLS algorithm }
\end{aligned}
$$

Vector $\bar{v}_{1}$ (error in raw count rate)

$$
\begin{aligned}
{\left[\bar{v}_{1}\right]_{\mathfrak{i}} } & =R_{\mathfrak{j}} / L \\
L & =\text { instrument live time for present sample }
\end{aligned}
$$

Vector $\bar{v}_{2}$ (error in instrument background count rate)

$b_{j} / L$ for single record reduced assays $(L=$ sample counting interval)

$\left[\bar{V}_{2}\right]_{j}=e^{2}\left(b_{i}\right)$ as returned from MLS algorithm for statistical analysis assays

Vector $\bar{v}_{4}$ (error in airborne ${ }^{214} \mathrm{Bi}$ count rate)

$$
\begin{aligned}
{\left[v_{4}\right]_{2} } & =a_{2}^{2}\left[e^{2}(u) / u^{2}+e^{2}(v) / v^{2}\right) \\
u & =G^{2 \pi}-f(H) G^{4 \pi} \\
v & =g(H)-0.19 f(H) \\
& e(u) \text { numerically computed from MLS algorithm } \\
& e(v) \text { numerically computed from MLS algorithm }
\end{aligned}
$$

Figure 5. Functional Form of Errors in Error Propagation Equation 
The specific detail for computing uncertainties in each error propagation step is described as appropriate throughout this specification.

\subsection{A DISCUSSION OF PRECISION}

It should be noted that equation (5.1) estimates only the precision error in the reduction equation. Precision-error estimates must not be misinterpreted as absolute errors. Absolute error is an unpredictable mixture of imprecisions and inaccuracies involved in both data collection and reduction.

\subsection{CALIBRATION}

Calibration constants for the reduction equation and related errors for the error propagation equation are derived from acquiring and analyzing calibration data over the following three calibration areas:

1. High-altitude flights over a large body of water.

2. Walker Field calibration pads.

3. Lake Mead Dynamic Test Range flights.

This paragraph specifies data acquisition, BFEC delivery requirements, and analysis procedures that were required by each subcontract work statement.

\subsection{CALIBRATION DATA ACQUISITION}

Precisely as configured for a NURE survey, radiometric data shall be recorded in compliance with Specification BFEC 1200-C and as described in the following paragraphs.

\subsubsection{High-Altitude Calibration}

High-altitude readings at five or more constant elevations shall be conducted beyond the U.S. continental shoreline. All possible precautions shall be observed to calibrate under atmospheric conditions conducive to the absence of airborne $214_{\mathrm{Bi}}$. A guide to minimum mean sea level (MSL) elevations is as follows:

- Fixed-wing aircraft: 1800, 2400, 3000, 3600, and 4500 meters.

- Rotary-wing aircraft: $1000,1500,2000,2500$, and 3400 meters. 
A minimum of 10 minutes of data shall be collected at each elevation. Aircraft altitude at each elevation shall not deviate by more than 40 meters. All ancillary data including accurate barometric pressure and air temperature readings shall be recorded as for NURE surveys. Accurate radar altimeter readings are not necessary. Spectrometer accumulation intervals shall be 1 second and 5 seconds for the $4 \pi$ and $2 \pi$ detector systems, respectively.

\subsubsection{Walker Field Pad Calibration}

Measurements shall be made with each detector subpackage centered over each of the five Walker Field calibration pads. Repeat measurements shall be made over Pad 1, the background pad. A $4 \pi$ system detector subpackage shall be appropriately defined in writing by the BFEC subcontract monitor prior to making these measurements. Two-pi detector readings shall be recorded for each $4 \pi$ subpackage reading. Accurate ancillary measurements shall be recorded for each $4 \pi$ subpackage measurement. A minimum of 120 seconds of data shall be acquired for each subpackage measurement, and spectrometer accumulation intervals shall be 1 second and 5 seconds for the $4 \pi$ and $2 \pi$ detector systems, respectively.

Subcontractors shall notify the BFEC subcontract monitor of the intended date and time for the Walker Field pad calibration sequence. Calibration shall not be attempted while any pad is visibly wet.

\subsubsection{Lake Mead Dynamic Test Range Calibration}

The location and physical layout of the Lake Mead Dynamic Test Range (DTR) are described in an earlier report (Geodata International, Inc., 1977). The test line is set as a north-northeast flight track 15.4 kilometers long extending $2.9 \mathrm{kilometers}$ inland from the edge of Lake Mead and $12.5 \mathrm{kilometers}$ over water.

The over-water portion of the test 1 ine extends $\mathrm{N} .12^{\circ} \mathrm{E}$. directly from the final DiR on-land marker to at least lat $36.1500^{\circ} \mathrm{N}$. Data collected over water between lat $36.0800^{\circ} \mathrm{N}$. and lat $36.1500^{\circ} \mathrm{N}$. shall be used for over-water calibration data analysis.

The over-land segment of the test line is identified by four markers, two triangles and two crosses. The two extreme outer markers locate the ends of the on-land flight track. Only data collected between the inner two triangular markers shali be used for over-land calibration data analysis. 
Unless otherwise agreed to by $B F E C$, the subcontractor shall provide at least 2 weeks advance notice to BFEC of the desired date for flying the Lake Mead Dynamic Test Range. The BFEC subcontract monitor will verify the acceptability of the date, provide final instructions, and provide ground monitoring as required. Lake Mead flights shall not be attempted within 4 days after any precipitation or during periods of no detectable breeze ( $<5$ knots).

During Lake Mead overflights, the total system shall be operated in accordance with Specification BFEC $1200-C$, flown consistent with normal survey procedures and at normal survey ground speed. The designated flight line shall be flown four times at each of the following altitudes: $61,91,122,152,183$, 213, 244, and 305 meters. Maximum deviations from specified terrain clearances shall not be greater than 15 meters. Spectrometer accumulation interval for the $4 \pi$ detector system shall be 1 second and for the $2 \pi$ detector system, 5 seconds.

\subsection{CALIBRATION DATA REPORTING REQUIREMENTS}

One report and ancillary data tapes shall be submitted to the BFEC subcontract monitor. The report shall contain the following sections:

1. Front cover information to include:

a. Report Title

b. Subcontractor's Name

c. Aircraft Type and Registry Number

d. Calibration Date

e. Report Date

2. Data acquisition system information to include:

a. Gamma-Ray Spectrometer

b. NaI Crystal Description, $4 \pi$ and $2 \pi$ Systems

c. Magnetometer

d. Radar Altimeter

e. Barometric Altimeter

f. Outside Air Temperature Monitor

g. Digital Recording System

h. Track Recovery System

i. Detector Heights Above Ground Level

3. Caibration summary information to include:

a. Dates Calibration Data Were Collected 
b. Location of High-Altitude Flights

c. Weather Conditions at Each Calibration Site

4. Raw spectral data tape flight-line information to include:

a. Flight-Line Numbers and Corresponding MSL Altitudes

for High-Altitude Flights

b. Flight-Line Numbers and Corresponding Terrain Clearances for Lake Mead Flights

c. Flight-Line Numbers and Corresponding Pad/Crystal Configuration for Walker Field Pad Calibration

Raw spectral data tapes ( 3 tapes) with calibration data recorded also shall be generated in accord with Specification BFEC 1200-C and delivered to BFEC. All calibration data will then be analyzed by BFEC to determine the following:

- That the subcontractor's system meets relevant BFEC specifications.

- All calibration coefficients and related uncertainties to be used by the subcontractor in the reduction and error propagation equations.

Within 30 days of receipt of report and tapes, BFEC will furnish the subcontractor with all calibration data analysis results. Problems associated with calibration will be reported to the subcontractor immediately after being detected by BFEC.

\subsection{CALIBRATION DATA ANALYSIS}

The following paragraphs detail all calibration data analys is routines that BFEC will perform to derive calibration constants and associated precision errors. Spectrometer calibration procedures and quality control checks are also described.

\subsubsection{Notation}

$$
\begin{aligned}
I_{j k} & =\text { counts observed in } k^{\text {th }} \text { spectrometer channel and } j^{\text {th }} \text { observation } \\
L_{j} & =\text { spectrometer live time for } j \text { th observation (seconds) } \\
A_{j} & =\text { aircraft terrain clearance for } j^{\text {th }} \text { observation (meters) } \\
T_{j} & =\text { ambient air temperature for } j \text { th observation }\left({ }^{\circ} \mathrm{C}\right. \text { ) } \\
P_{j} & =\text { ambient air pressure for } j \text { th observation (mm } \mathrm{Hg} \text { ) } \\
E_{i}^{U}, E_{i}^{\ell} & =\text { upper and lower energy } 1 \text { imits for } i \text { th window (keV) }
\end{aligned}
$$




$$
\text { subscript } i=\left\{\begin{array}{l}
1=\text { measurement relative to } 40_{K} \text { activity } \\
2=\text { measurement relative to } 214 \mathrm{Bi} \text { activity } \\
3=\text { measurement relative to } 208 \mathrm{Tl} \text { activity } \\
4=\text { measurement relative to total activity } \\
5=\text { measurement relative to cosmic activity }
\end{array}\right.
$$

subscript $\mathrm{j}=$ observation

subscript $k=$ observation within a data record or system subpackage superscript $4 \pi, 2 \pi=$ terrestrial and atmospheric spectrometer systems, respectively. Omitted superscripts identifying spectrometer system signify operations are identical for both.

\subsubsection{Processing to Raw Window Count Rates}

Energy window boundaries for detection of $40_{\mathrm{K}},{ }^{214} \mathrm{Bi},{ }^{208} \mathrm{Tl}$, total, and cosmic activity for the $4 \pi$ detector system are given in Table 1 . Spectrometer channels $\left(\mathrm{CH}_{j}^{u}, \mathrm{CH}_{j}^{l}\right)$ corresponding to $\left(E_{j}^{u}, E_{j}^{l}\right)$ are computed by assuming a linear relationship between energy and channel numbers. This relationship in continuous form is

$$
E=E_{0}+\Delta E \cdot C H
$$

The channel and fraction thereof corresponding to a preset energy is therefore

$$
C H=\left(E-E_{0}\right) / \Delta E
$$

The constants $\left(E_{0}, \Delta E\right)$ are termed spectrometer energy calibration constants. These constants, $E_{0}$ and $\Delta E$, are calculated using averaged $4 \pi$ and averaged $2 \pi$ spectral shapes from which known and recognizable photopeaks are fit to a Gaussian shape to locate photopeak centroid channels. For survey data, one contiguous flight line or flight-line segment is summed to generate averaged $4 \pi$ and $2 \pi$ spectral shapes, and the process repeated for each flight line or flight-line segment. For calibration data, all flight lines within each calibration site (i.e., high altitude, Walker Field pads, Lake Mead) are used to generate $4 \pi$ and $2 \pi$ spectral shapes. 
Table 1. Energy Limits for Spectral Windows

\begin{tabular}{|c|c|c|c|c|c|}
\hline $\begin{array}{l}\text { Spectral } \\
\text { Window }\end{array}$ & $\begin{array}{l}\text { Emission } \\
\text { Line (keV) }\end{array}$ & $\begin{array}{l}\text { Subscript } \\
\text { Index }\langle i\rangle\end{array}$ & $E^{u}(k e V)$ & $E^{l}(\mathrm{keV})$ & $\Delta E(\mathrm{keV})$ \\
\hline $40_{k}$ & 1461 & 1 & 1561 & 1361 & 200 \\
\hline $214_{B i}$ & 1764 & 2 & 1864 & 1664 & 200 \\
\hline${ }^{208} \mathrm{TT}$ & 2615 & 3 & 2815 & 2415 & 400 \\
\hline Total & - & 4 & 3000 & 400 & 2600 \\
\hline Cosmic & - & 5 & 6000 & 3000 & 3000 \\
\hline
\end{tabular}

The recognizable photopeaks used for $\left(E_{0}, \Delta E\right)$ definition are given in Table 2. The numerical method used to fit a Gaussian shape to each photopeak is described in Appendix $A$ of this report. Spectrometer calibration quality control checks that are performed on each averaged spectrum are described in paragraph 6.3.2.1.

As described in Appendix $A$, output from repetitive application of the photopeak fitting process are $\left[\mathrm{CH}_{i}^{c}, e\left(\mathrm{CH}_{j}^{c}\right)\right]$ for $i=1,2,3$. These quantities are the channel centroid positions and related errors for each photopeak.

Table 2. Photopeaks for $E_{0}, \Delta E$ Definition

\begin{tabular}{rcc}
\hline Photopeak & $\begin{array}{c}\text { Emission Line } \\
(\mathrm{keV})\end{array}$ & $\begin{array}{c}\text { Error } \\
(\mathrm{keV})\end{array}$ \\
\hline $\begin{array}{r}40_{\mathrm{K}} \\
214 \mathrm{Bi}\end{array}$ & 1460.85 & 0.10 \\
$208 \mathrm{Tl}$ & 1764.49 & 0.07 \\
\hline
\end{tabular}

Letting $E_{i}^{c}$ represent the corresponding emission line for $\mathrm{CH}_{j}^{\mathrm{C}}$ given in Table 2, $E_{0}$ and $\Delta E$ are calculated for each spectrometer system by assigning

$$
\begin{aligned}
X_{i} & =\mathrm{CH}_{i}^{C} \\
e\left(X_{i}\right) & =e\left(\mathrm{CH}_{i}^{C}\right)
\end{aligned}
$$




$$
\begin{aligned}
Y_{i} & =E_{i}^{c} \\
e\left(Y_{j}\right) & =e\left(E_{i}^{c}\right)
\end{aligned}
$$

and solving the resulting set of equations posed by the multiple least squares (MLS) regression analysis described in Appendix $B$.

Output from the MLS regression analys is are numerical values for $\left(E_{0}, \Delta E\right)$. Spectrometer channels and fractions thereof corresponding to $\left(E_{i}^{\ell}, E_{j}^{u}\right)$ are then computed using equation (6.2) and the energy window limits given in Table 1.

Raw window count rates for the $j^{\text {th }}$ observation and $i^{\text {th }}$ window are computed from the equation

where

$$
R_{i j}=S_{i j} / L_{j}
$$

$$
S_{i j}=\Sigma_{k=K 1}^{K 2}\left(I_{j k}\right)+\Delta_{1 i} I_{j}(K 1-1)+\Delta_{2 i} I_{j(K 2+1)}
$$

and

$$
\begin{aligned}
\mathrm{K} 1 & =\text { integral part of }\left(\mathrm{CH}_{j}^{\ell}+1\right) \\
\mathrm{K} 2 & =\text { integral part of }\left(\mathrm{CH}_{i}^{u}-1\right) \\
\Delta_{1 i} & =\mathrm{K} 1-\mathrm{CH}_{i}^{\ell} \\
\Delta_{2 i} & =\mathrm{CH}_{i}^{u}-(\mathrm{K} 2+1)
\end{aligned}
$$

Precision errors in $R_{\mathbf{i} j}$ are approximated from the equation

$$
e^{2}\left(R_{i j}\right)=R_{i j} / L_{j}
$$

Equation (6.5) assumes observed channel counts obey Poisson counting statistics.

For processing single record reduced data, $R_{i j}$, as computed by equation (6.3), for each $j$, is placed in the vector $R_{1}$ in the reduction equation, and $e^{2}\left(R_{i j}\right)$, as computed by equation $(6.5)$, placed in the vector $\bar{v}_{1}$ in the error propagation equation.

5.3.2.1 Spectrometer Calibration Quality Control Checks - Quantities needed to determine gain and energy-resolution characteristics of the $4 \pi$ and $2 \pi$ spectrometers $\left(E_{0}, \Delta E\right)$ are output from the photopeak fitting process and calibration. In addition, the fitting process yields the following: 


$$
\begin{aligned}
s_{i}= & \text { Gaussian spread for } i^{\text {th }} \text { photopeak } \\
& \text { (computed according to Appendix A) } \\
\mathrm{CH}_{i}^{\mathrm{C}}= & \text { channel centroid for } i^{\text {th }} \text { photopeak } \\
E_{i}^{\prime}= & \text { calculated emission line energy for } i^{\text {th }} \text { photo- } \\
& \text { peak }\left(E_{i}^{\prime}=E_{0}+\Delta E \cdot C H_{i}^{c}\right)
\end{aligned}
$$

Full-width-half-maximum (FWHM) energy resolution for the $i^{\text {th }}(i=1,2,3)$ photopeak in percent is calculated from the equation

$$
\% \text { WHM }_{i}=235 s_{i} \Delta E / E_{i}^{C}
$$

All such energy-resolution calculations shall be equal to or below those values required in paragraph 3.1.2, Specification BFEC 1200-C.

Spectrometer gain linearity $(G L)$ for $i=1,2,3$ is computed from the equation

$$
\% G L_{i}=100 E_{i}^{\prime} / E_{i}^{C}
$$

Computed $\%_{G_{i}}$ shall be within 1 percent of 100 percent.

Spectrometer differential linearity characteristics are determined by visually inspecting average spectral shapes. Any obvious spikes in channel intensities shall be noted and brought to the attention of the BFEC subcontract monitor.

Problems noted with FWHM energy resolutions, gain linearity, or differential linearity shall be sufficient cause to disqualify the survey data or calibration data from which these calculations were derived.

\subsubsection{High-Altitude Calibration Analysis}

Analysis of high-altitude data, acquired as specified in paragraph 6.1.1, yields estimates of the following:

- Instrument background intensities in each $4 \pi$ and $2 \pi$ window.

- Cosmic continuum stripping ratios for each $4 \pi$ and $2 \pi$ window. Input to this analysis are as follows:

$$
\begin{aligned}
\bar{R}_{i j} & =\text { averaged raw window count rate for } j^{\text {th }} \text { altitude and } i^{\text {th }} \text { window } \\
e\left(\bar{R}_{i j}\right) & =\text { error in } \bar{R}_{i j}
\end{aligned}
$$


Determination of averaged $r a w$ window count rates, associated uncertainties, and the Poisson count-rate quality check for high-altitude $\vec{R}_{i j}$ is described in Appendix C.

Repeated high-altitude observations have shown that, in the absence of an airborne $214_{B i}$ signal, there exists a linear relationship between any particular raw window count rate and the cosmic window count rate. Moreover, this linear dependence is quite insensitive to MSL altitude, latitude, or longitude. The linear relationship assumed is

where

$$
\bar{R}_{i j}=-s_{i 5} \bar{R}_{5 j}+b_{i}
$$

$$
\begin{aligned}
s_{i 5} & =\text { cosmic stripping ratio for } i^{\text {th }} \text { window } \\
b_{j} & =\text { instrument background for } i^{\text {th }} \text { window }
\end{aligned}
$$

These calibration constants are numerically determined for each $4 \pi$ and $2 \pi$ window by fixing $i$, assigning

$$
\begin{aligned}
x_{j} & =\bar{R}_{5 j} \\
e\left(x_{j}\right) & =e\left(\bar{R}_{5 j}\right) \\
Y_{j} & =\bar{R}_{i j} \\
e\left(Y_{j}\right) & =e\left(\bar{R}_{i j}\right)
\end{aligned}
$$

and solving the resultant equations as posed by the MLS regression algorithm given in Appendix $B$.

Output from the MLS regression analysis are the calibration constants $\left[b_{i}, e\left(b_{i}\right)\right]$ and $\left[s_{i 5}, e\left(s_{i 5}\right)\right]$ for each $4 \pi$ and $2 \pi$ window. Instrument backgrounds and cosmic stripping ratios are considered consistent (i.e., uncontaminated by a nonuniforn distribution of airborne $214_{B i}$ ) if for all ij

$$
\left|\bar{R}_{i j}-\bar{R}_{i j}^{\prime}\right| \leq 3\left[e^{2}\left(b_{i}\right)+\bar{R}_{5 j}^{2} e^{2}\left(s_{i 5}\right)+s_{i 5}^{2} e^{2}\left(\bar{R}_{5 j}\right)\right]^{1 / 2}
$$

where $\bar{R}_{i j}^{\prime}$ are the adjusted mean count rates as determined from equation (B.16), Appendix B. Equation (6.9) states that the difference between average observed raw window count rates and the MLS line shall be within three times the error involved in computing the MLS line.

Provided tnat both the consistency checks in equation (5.9) and the absolute calibration checks given in paragraph 5.3.5.1 are met, calibration 
constants $\left[b_{i}, e^{2}\left(b_{i}\right)\right]$ and $\left[s_{i 5}, e^{2}\left(s_{i 5}\right)\right]$ are appropriately placed in the reduction and error propagation equations. For processing single record data, the value for $e^{2}\left(b_{j}\right)$ is calculated from the equation

$$
e^{2}\left(b_{i}\right)=b_{i} / L_{j}
$$

For processing statistical analysis data, the value for $e^{2}\left(b_{j}\right)$ is the square of the value returned from the MLS regression analysis.

\subsubsection{Walker Field Pad Calibration Analysis}

Analysis of Walker Field pad calibration data yields estimates of zeroaltitude Compton-stripping coefficients. Stripping constants remove the composite effect of Compton down-scattering and multiple-source interference between windows. Multiple-source interference arises from the fact that gamma rays emitted from different sources are present in each window. For example, note the ${ }^{214_{B i}} 2.448-M e V$ line present in the ${ }^{208} \mathrm{Tl}$ window. Quantitatively Compton scattering dominates these two effects, and thus the nomenclature "Compton stripping."

Walker Field pad calibration data are also analyzed to estimate a system's $2 \pi$ detector shielding efficiency. Shielding efficiency is a measure of the volume-ratioed amount of ground radiation entering the $2 \pi$ detector system. Shielding efficiency computations lead to a quantitative means of removing the ground contribution when estimating the airborne ${ }^{214} \mathrm{Bi}$ correction from $2 \pi$ detector data.

Input to Walker Field pad data analysis are as follows:

$$
\begin{aligned}
& \vec{R}_{i j k}^{4 \pi}=\begin{array}{l}
\text { average } 4 \pi \text { count rate for } j^{\text {th }} \text { pad, } i^{\text {th }} \text { window, and } k^{\text {th }} \\
\text { detector subpackage }
\end{array} \\
& e\left(R_{i j k}^{4 \pi}\right)=\text { error in } \vec{R}^{4}{ }^{\pi} j k \\
& C_{i j}=\text { assigned element concentration for } j^{\text {th }} \text { pad and } i^{\text {th }} \text { radioelement } \\
& e\left(C_{i j}\right)=\text { error in } C_{i j}
\end{aligned}
$$

Determination of averaged Walker Field pad count-rate quantities and performance of quality control checks are accompl ished in accord with paragraph 
6.3.2 and Appendix C. Pad concentrations and related errors to be used in this analysis are given in Table 3.

Table 3. Walker Field Pad Concentrations ${ }^{a}$

\begin{tabular}{|c|c|c|c|}
\hline Pad & & Concentrati & \\
\hline No. & $\% \mathrm{~K}$ & $\mathrm{ppm} \mathrm{eU}$ & ppm eTh \\
\hline 1 & $1.45 \pm 0.01$ & $2.2 \pm 0.1$ & $6.3 \pm 0.1$ \\
\hline 2 & $5.14 \pm 0.05$ & $5.1 \pm 0.2$ & $8.5 \pm 0.2$ \\
\hline 3 & $2.01 \pm 0.02$ & $5.1 \pm 0.1$ & $45.3 \pm 0.4$ \\
\hline 4 & $2.03 \pm 0.02$ & $30.3 \pm 1.0^{b}$ & $9.2 \pm 0.2$ \\
\hline 5 & $4.11 \pm 0.03$ & $20.4 \pm 1.0^{b}$ & $17.5 \pm 0.2$ \\
\hline
\end{tabular}

a Reference: Ward, D. L., Construction of Calibration Pads Facility, Walker Field, Grand Junction, Colorado: U.S. Department of Energy Open-File Report GJBX-37(78).

$b_{M L S}$ regression analysis consistently has shown a precision error of approximately $1 \mathrm{ppm}$ eU and eTh, respectively.

Total $4 \pi$ system raw window count rates and uncertainties are computed by

and

$$
\bar{R}_{i j}^{4 \pi}=\Sigma_{k=1}^{K}\left(\bar{R}_{i j k}^{4 \pi}\right)
$$

$$
e^{2}\left(\bar{R}_{i j}^{4 \pi}\right)=\sum_{k=1}^{K}\left[e^{2}\left(\bar{R}_{i j k}^{4 \pi}\right)\right]
$$

where $K$ denotes the total number of subpackages comprising the $4 \pi$ system.

Having averaged total system count rates, the next step is to ensure that background levels remained constant during the entire calibration process. This is necessary because analysis of Walker Field pad calibration data assumes a constant background. Variation in background is detected by comparing the two measurements taken over Pad 1, the background pad. To ensure ininimal variation in backgrounds, all averaged $4 \pi$ and $2 \pi$ raw window count rates for Pad 1 must be within one standard error of the other. 
Determination of total $4 \pi$ system sensitivities relative to assigned pad concentration is the first analysis step. System sensitivities are determined by assuming a linear relationship between averaged raw window count rates and assigned pad concentrations. This is to say, for each pad and each window there exist coefficients $a_{i k}$ and $b_{i}$ such that

$$
\bar{R}_{i j}^{4 \pi}=\Sigma_{k=1}^{3}\left(a_{i k} c_{k j}\right)+b_{i}
$$

Constants $a_{i k}$ and $b_{j}$ are determined for each $i$ by assigning

$$
\begin{aligned}
x_{k j} & =C_{k j} \\
e\left(x_{k j}\right) & =e\left(C_{k j}\right) \\
Y_{j} & =\bar{R}_{i j}^{4 \pi} \\
e\left(Y_{j}\right) & =e\left(R_{i j}^{4 \pi}\right)
\end{aligned}
$$

and solving the resultant set of equations posed by the MLS regression algorithm given in Appendix $B$.

As returned from the MLS regression analysis, the constants $a_{i j}$ are zeroaltitude walker field pad sensitivities relative to the $i^{\text {th }}$ radioelement. System sensitivities are approximately normalized to infinite-plane sensitivities using the equation

where

$$
k_{i}^{W F}=a_{i j} / p(H)
$$

$$
\begin{aligned}
p(H)= & \text { scale factor taken from the abscissa with ordinate } H \text { given } \\
& \text { in Figure } 6 \\
H= & \text { detector height above ground level (meters) }
\end{aligned}
$$

Zero-altitude, infinite-plane system sensitivities shall be compared with approximate downward-continued 122 -meter Lake Mead system sensitivities. This comparison is described in paragraph 6.3.5.3.

Compton-stripping coefficients are computed by assuming a linear relationship between pad radioelement concentrations and observed window count rates. For all pads and radioelement windows, pad concentrations are converted to equivalent window count rates using the equation

$$
c_{i j}^{i}=a_{i j} c_{i j}
$$




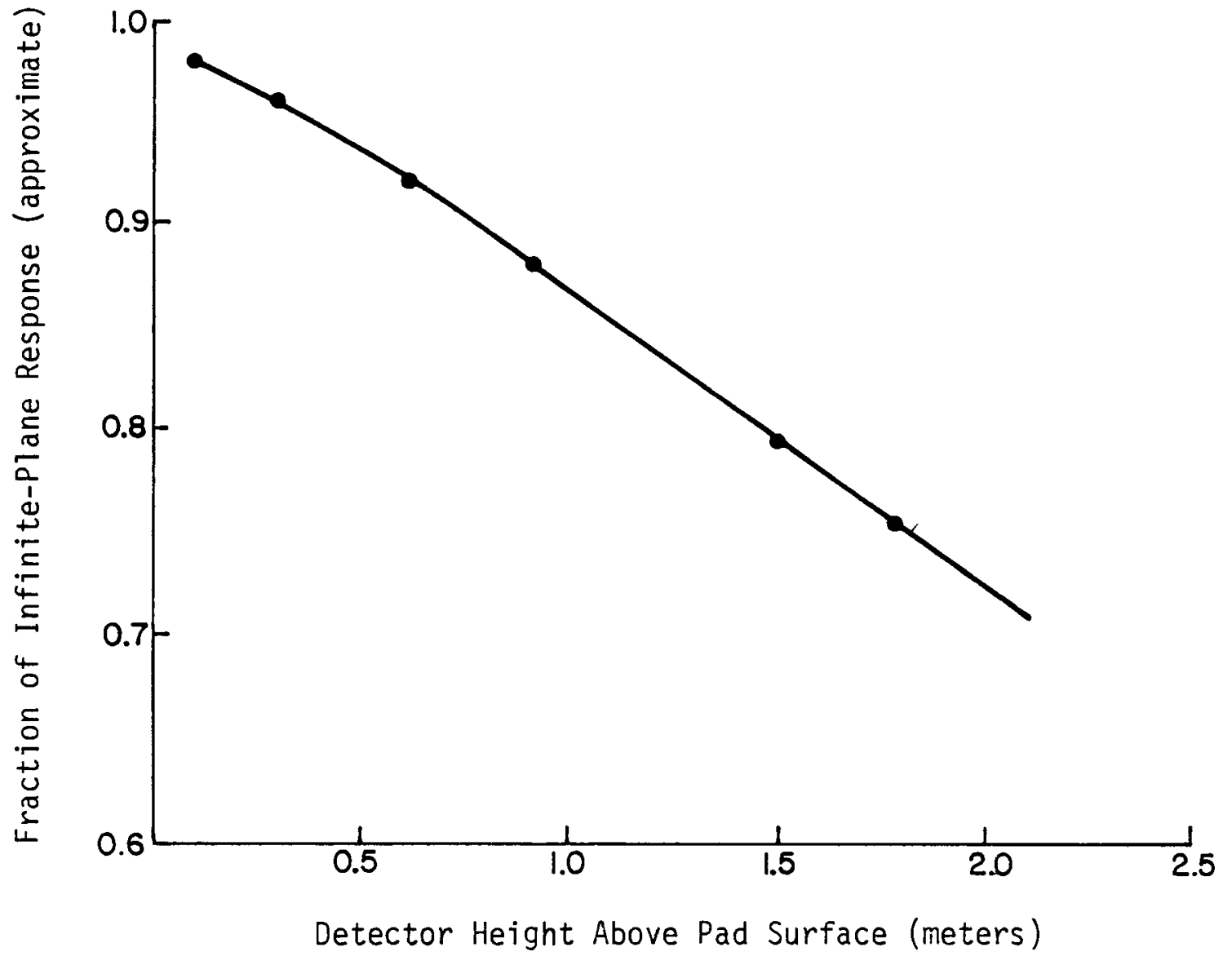

Figure 6. Walker Field Pad Infinite-Plane Normalization Curve (approximate) 
Errors in $C_{i j}^{\prime}$ are determined from the equation

$$
e^{2}\left(C_{i j}^{\prime}\right)=a_{i j}^{2} e^{2}\left(C_{i j}\right)+c_{i j}^{2} e^{2}\left(a_{i j}\right)
$$

Differences between observed window count rates and equivalent window count rates are attributable to Compton scattering, interfering photopeaks, and an assumed constant background. Letting

and

$$
\Delta R_{i j}=C_{i j}^{\prime}-R_{i j}^{4 \pi}
$$

$$
e^{2}\left(\Delta R_{i j}\right)=e^{2}\left(C_{i j}^{\prime}\right)+e^{2}\left(\bar{R}_{i j}^{4 \pi}\right)
$$

the linear relationship assumed between $\Delta R_{i j}$ and $R_{i j}^{4 \pi}$ is

$$
\Delta R_{i j}=\sum_{\substack{k=1 \\ k \neq j}}^{3}\left(s_{i k} \bar{R}_{k j}^{4 \pi}\right)+b_{i}
$$

Stripping constants $s_{i k}(i=1,2,3 ; k=1,2,3 ; k \neq i)$ are computed by fixing $i$ and assigning

$$
\begin{aligned}
x_{k j} & =\bar{R}_{k j}^{4 \pi} \\
e\left(X_{k j}\right) & =e\left(\bar{R}_{k j}^{4 \pi}\right) \\
Y_{j} & =\Delta R_{i j} \\
e\left(Y_{j}\right) & =e\left(\Delta R_{i j}\right)
\end{aligned}
$$

and solving the resultant set of equations posed by the MLS regression algorithm given in Appendix $B$.

Output from the MLS regression algorithm are $s_{i k}$ and $e\left(s_{i k}\right)$. The constants $s_{21}$ and $s_{31}$ are a measure of system pulse-pair pile-up which, in a survey environment, are assumed negligib?e. Thus, they are set to zero in the reduction equation. Stripping coefficients $\mathrm{s}_{12}, \mathrm{~s}_{13}, \mathrm{~s}_{23}$, and $\mathrm{s}_{32}$ are placed in their appropriate positions in the reduction equation, and $\mathrm{e}^{2}\left(\mathrm{~s}_{12}\right)$, $\mathrm{e}^{2}\left(\mathrm{~s}_{13}\right), \mathrm{e}^{2}\left(\mathrm{~s}_{23}\right)$, and $\mathrm{e}^{2}\left(\mathrm{~s}_{32}\right)$ placed in their appropriate positions in the error propagation equation. 
Averaged $4 \pi$ and $2 \pi$ Walker Field pad raw window count rates are combined with Lake Mead land/water data to model and correct for ground-signal leakage into the $2 \pi$ detector system. This procedure is described in paragraph 6.3.5.2.

\subsubsection{Lake Mead Dynamic Test Range Calibration Analys is}

Analysis of Lake Mead calibration data determines calibration constants for the following:

- Correction for airborne $214_{B i}$.

- Window count-rate attenuation as a function of terrain clearance.

- 122-meter system sensitivities.

In addition, Lake Mead over-water count rates provide an independent validation for instrument and cosmic backgrounds as derived from high-altitude calibration flights.

Input to the Lake Mead calibration data analysis are as follows:

$$
\begin{aligned}
\bar{R}_{i j k}= & \text { averaged } 4 \pi \text { and } 2 \pi \text { system raw window count rate for } \\
& j \text { th } f \text { light line, } i \text { th window, and } k \text { th flight-line segment } \\
e\left(\bar{R}_{i j k}\right)= & \text { uncertainty in } \bar{R}_{i j k} \\
\bar{P}_{j k}= & \text { average outside air pressure for } j^{\text {th }} \text { flight line and } \\
& k \text { th } f l \text { ight-line segment } \\
\bar{T}_{j k}= & \text { average outside air temperature for } j \text { th flight line } \\
& \text { and } k \text { th flight-line segment } \\
\bar{A}_{j k}= & \text { average radar altitude (terrain clearance) for } j \text { th } \\
& \text { flight line and } k \text { th flight-line segment } \\
e\left(\bar{A}_{j k}\right)= & \text { uncertainty in } \bar{A}_{j k} \\
\bar{R}_{i j}= & \text { averaged } 4 \pi \text { and } 2 \pi \text { system raw window count rate for } j \text { th } \\
& \text { walker Field calibration pad and } i \text { th window } \\
e\left(\bar{R}_{i j}\right)= & \text { uncertainty in } \bar{R}_{i j}
\end{aligned}
$$

For Lake Mead data, the third subscript $k$ is given to mean

$$
k=\left\{\begin{array}{l}
1 \Leftrightarrow \text { land segment of each flight line } \\
2 \Leftrightarrow \text { water segment of each flight line }
\end{array}\right.
$$


Land/water segments, from which average count rates are computed, are positionally defined along the standard flight line as

$$
\begin{aligned}
\text { land } & \Leftrightarrow 36.0131^{\circ} \leq \text { latitudes } \leq 36.0272^{\circ} \\
\text { water } & \Leftrightarrow 36.0800^{\circ} \leq \text { latitudes } \leq 36.1500^{\circ}
\end{aligned}
$$

6.3.5.1 Instrument and Cosmic Background Validation - The first analysis procedure for Lake Mead data is instrument and cosmic background validation. This validation requires average Lake Mead over-water count rates to be processed through reduction step (3) and errors propagated through error propagation step (3).

Referring to the reduction and error propagation equations and letting $\vec{R}(3)$ and $\bar{E}(3)$ denote resultant count rates and related errors through reduction step (3), expressions for $\bar{R}^{-(3)}$ and $\bar{E}^{(3)}$ are

$$
\bar{R}^{(3)}=S(\bar{R}-\bar{B})
$$

and

$$
\bar{E}(3)=M_{2}\left(\bar{V}_{1}+\bar{V}_{2}\right)+M_{3} \bar{V}_{3}
$$

Using calibration constants and associated errors determined from paragraphs 6.3.3 and 6.3.4 (as applied to statistical analysis reduction) and using $R_{i j 2}^{4 \pi}$ and $e\left(\bar{R}_{i j 2}^{4 \pi}\right)$ as input to equations (6.20) and (6.21), all over-water flightline data shall be reduced and error propagated through reduction step (3). Instrument and cosmic backgrounds shall be considered accurate for $i=1$ and $i=3\left({ }^{40} \mathrm{~K}\right.$ and ${ }^{208} \mathrm{Tl}$ window $)$ if

$$
\left|\left[\bar{R}^{(3)}\right]_{j}\right| \leq 3\left\{\left[\bar{E}^{(3)}\right]_{i}\right\} 1 / 2
$$

Equation (6.22) states that residual over-water count rates, after stripping, nust be within three standard errors of zero. Failure to pass this test for all Lake Mead over-water flight-line segments is sufficient cause to require an additional high-altitude calibration. 


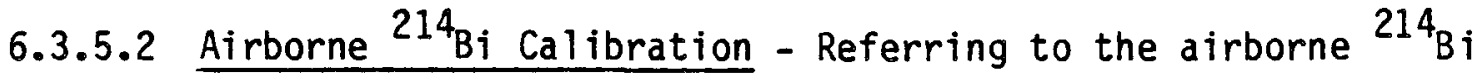

correction expression in the reduction equation, values for two calibration functions are needed. These are

$$
\begin{aligned}
& f(H)=2 \pi \text { effective shielding efficiency function } \\
& g(H)=2 \pi-t 0-4 \pi \text { coupling function }
\end{aligned}
$$

Reasonable estimates for the constants in $f(H)$ and $g(H)$ require that the concentration and spatial distribution of airborne $214_{B i}$ on the day of the Lake Mead over-flights satisfy the following two conditions:

1. A concentration strength sufficiently large to be precisely determined.

2. A uniform spatial distribution between land and water.

Past experience has shown that these two conditions are usually met in the spring and early summer seasons of the year, if sorties are flown before midday and if a detectable breeze ( $>5$ knots) is present. This specification qualifies the circumstances under which an inaccurate airborne ${ }^{214} \mathrm{Bi}$ results. If an invalid condition arises, the BFEC subcontract monitor will decide and direct the appropriate corrective action.

Shielding efficiencies for windows $i=1,2,3,4$ are assumed to be 1 inear functions of standard temperature and pressure (STP) altitude. (A linear function is a first-order approximation to an exponential function.) This is expressed in equation form as

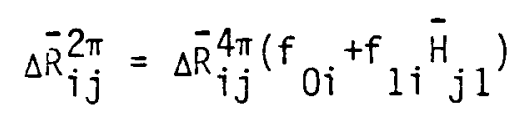

where for both $4 \pi$ and $2 \pi$ count rates

$$
\Delta \bar{R}_{i j}=\bar{R}_{i j 1}-\bar{R}_{i j 2} \text { (1 and minus water) }
$$

and

$$
e^{2}\left(\Delta \bar{R}_{i j}\right)=e^{2}\left(\bar{R}_{i j 1}\right)+e^{2}\left(\bar{R}_{i j 2}\right)
$$

Average STP altitudes $\bar{H}_{j k}$ in equation (6.23) are computed by

$$
\bar{H}_{j k}=273 \bar{A}_{j k} \bar{p}_{j k} /\left[760\left(273+\bar{T}_{j k}\right)\right]
$$


and uncertainties in Lake Mead STP altitudes are computed by

$$
e\left(\bar{H}_{j k}\right)=273 e\left(\bar{A}_{j k}\right) \bar{P}_{j k} /\left[760\left(273+\bar{T}_{j k}\right)\right]
$$

Walker Field pad count rates are simultaneously included in this determination by

$$
\Delta \bar{R}_{i j}=\bar{R}_{i j}-\bar{R}_{j l} \quad(j \neq 1)
$$

and

$$
\bar{H}_{j 1}, e\left(\bar{H}_{j 1}\right)=0
$$

Coefficients $\left(f_{0 i}, f_{1 i}\right)$ in equation (6.23) for each $i$ are computed by letting

$$
r_{i j}=\Delta \bar{R}_{i j}^{2 \pi} / \Delta \bar{R}_{i j}^{4 \pi}
$$

and

$$
e\left(r_{i j}\right)=r_{i j}\left[e^{2}\left(\Delta R_{i j}^{2 \pi}\right) /\left(\Delta R_{i j}^{2 \pi}\right)^{2}+e^{2}\left(\Delta R_{i j}^{4 \pi}\right) /\left(\Delta R_{i j}^{-4 \pi}\right)^{2}\right]^{1 / 2}
$$

and

$$
e^{2}\left(\Delta \vec{R}_{i j}\right)=e^{2}\left(\vec{R}_{i j 1}\right)+e^{2}\left(\bar{R}_{i j 2}\right)
$$

and assigning

$$
\begin{aligned}
x_{j} & =\bar{H}_{j 1} \\
e\left(x_{j}\right) & =e\left(\bar{H}_{j 1}\right) \\
Y_{j} & =r_{i j} \\
e\left(Y_{j}\right) & =e\left(r_{i j}\right)
\end{aligned}
$$

and solving the resultant equations posed by the MLS regression algorithm given in Appendix B. 
Output from the MLS regression algorithm are $\left[f_{0 i}, e\left(f_{0 i}\right)\right]$ and $\left[f_{1 i}, e\left(f_{1 i}\right)\right]$ for $i=1,2,3,4$. Survey-altitude (122-meter) shielding efficiencies in percent are computed from the equation

$$
\% \mathrm{SH}_{\mathbf{i}}=100\left[1-f_{i}(122) \mathrm{V}\right]
$$

where $V$ represents the $4 \pi-t 0-2 \pi$ crystal volume ratio. Uncertainty in $\% \mathrm{SH}_{j}$ is computed by

$$
e\left(\% \mathrm{SH}_{i}\right)=100 \mathrm{Ve}\left(\mathrm{f}_{0 j}\right)
$$

Shielding efficiencies for $i=1,2,3\left({ }^{40} \mathrm{~K}, 214_{\mathrm{B}} \mathrm{i},{ }^{208} \mathrm{Tl}\right.$ windows $)$ shall be within one standard error of the requirements stated in paragraph 3.2, Specification BFEC 1200-C.

Since the $2 \pi$ total-activity window $(i=4)$ is utilized for detection of airborne $214_{\mathrm{Bi}}$, the coefficients $\left[\mathrm{f}_{04}, \mathrm{e}\left(\mathrm{f}_{04}\right)\right]$ and $\left[\mathrm{f}_{14}, \mathrm{e}\left(\mathrm{f}_{14}\right)\right]$ are placed in their appropriate positions in the reduction and error propagation equations.

The second airborne ${ }^{214_{B i}}$ calibration function, $g(H)$, relates airborne $214_{B} i$ counts observed in the $2 \pi$ total-activity window to airborne ${ }^{214_{B}}$ i counts observed in the $4 \pi{ }^{214} B i$ window. This function, $g(H)$, is determined by subtracting instrument and cosmic backgrounds from both $2 \pi$ and $4 \pi$ over-water raw count rates, forming their ratio, and then assuming a linear altitude dependence (first-order approximation to an exponential function) between ratios and STP altitude. Let

with

$$
r_{j}=u_{j} / v_{j}
$$

and

$$
\begin{aligned}
& u_{j}=\bar{R}_{4 j 2}^{2^{\pi}}+s_{45}^{2 \pi-2 \pi}{ }_{5 j 2}^{2 \pi}-b_{4}^{2 \pi} \\
& v_{j}=\bar{R}_{2 j 2}^{-4 \pi}+s 5_{5 j 2}^{4 \pi-R^{4 \pi}}-b_{2}^{4 \pi}
\end{aligned}
$$

$$
e\left(r_{j}\right)=u_{j} / v_{j}\left[e^{2}\left(u_{j}\right) / u_{j}^{2}+e^{2}\left(v_{j}\right) / v_{j}^{2}\right]^{1 / 2}
$$


with

$$
\begin{aligned}
e^{2}\left(u_{j}\right) & =e^{2}\left(\bar{R}_{4 j 2}^{2 \pi}\right)+\left(s_{45}^{2 \pi}\right)^{2} e^{2}\left(\bar{R}_{5 j 2}^{2 \pi}\right)+\left(\bar{R}_{5 j 2}^{2 \pi}\right)^{2} e^{2}\left(s_{45}^{2 \pi}\right)+e^{2}\left(b_{4}^{2 \pi}\right) \\
e^{2}\left(v_{j}\right) & =e^{2}\left(\bar{R}_{2 j 2}^{4 \pi}\right)+\left(s_{25}^{4 \pi}\right)^{2} e^{2}\left(\bar{R}_{5 j 2}^{4 \pi}\right)+\left(\bar{R}_{5 j 2}^{4 \pi}\right)^{2} e^{2}\left(s_{25}^{4 \pi}\right)+e^{2}\left(b_{2}^{4 \pi}\right) \\
e^{2}\left(b_{4}^{2 \pi}\right) & =\text { square of value returned from MLS algorithm } \\
e^{2}\left(b_{2}^{4 \pi}\right) & =\text { square of value returned from MLS algorithm }
\end{aligned}
$$

The inear relationship assumed is

$$
r_{j}=g_{0}+g_{1} \bar{H}_{j 2}
$$

Coefficients $\left(g_{0}, g_{1}\right)$ are deternined by assigning

$$
\begin{aligned}
x_{j} & =\bar{H}_{j 2} \\
e\left(x_{j}\right) & =e\left(\bar{H}_{j 2}\right) \\
y_{j} & =r_{j} \\
e\left(Y_{j}\right) & =e\left(r_{j}\right)
\end{aligned}
$$

and solving the resulting equations as posed by the MLS regression analysis given in Appendix $B$.

Output from the MLS algorithm are $\left[g_{0}, e\left(g_{0}\right)\right]$ and $\left[g_{1}, e\left(g_{1}\right)\right]$. An accurate airborne ${ }^{214} \mathrm{Bi}$ correction requires that the coefficient of variation for $g$ (122) be known to a reasonable degree of precision. Therefore, the minimum coefficient of variation acceptable for $g(122)$ is 20 percent, or

$$
\mathrm{e}[\mathrm{g}(122)] / g(122) \leq 0.20
$$


Provided the inequality in equation (6.42) is met, $g(H)$ and $e[g(H)]$ are appropriately placed in the reduction and error propagation equations.

6.3.5.3 Altitude and System Normalization - Step (5) in the reduction equation involves two normalization corrections. The first normalization is an approximate analytic continuation of background- and Compton-corrected count rates to a constant datum. The constant datum is defined as a 122-meter uniform air column of STP density. The second nornalization involves dividing out 122-meter system sensitivities; this step is necessary to provide continuity with respect to magnitude among the several detection systems utilized in NURE aerial surveys.

Altitude attenuation and 122-meter STP system count rates are determined by assuming

$$
\Delta \bar{R}_{i j}^{S}=\bar{R}_{i} \exp \left[+\mu_{j}\left(122-\bar{H}_{j 1}\right)\right]
$$

where

$$
\begin{aligned}
\Delta R_{i j}^{S}= & \text { Compton-stripped, land-minus-water count rates for } \\
& j^{\text {th }} \text { altitude and } i^{\text {th }} \text { window }(i=1,2,3,4) \\
\bar{R}_{i}= & 122-\text { meter STP count rate for } i^{\text {th }} \text { window } \\
\mu_{j}= & \text { STP linear attenuation coefficient for } i^{\text {th }} \text { window }
\end{aligned}
$$

Being Compton-stripped count rates, $\Delta \vec{R}_{i j}^{s}$ has the form for $i=1,2,3$

$$
\Delta \bar{R}_{i j}^{S}=\Sigma_{k=1}^{3}\left(s_{i k} \Delta \bar{R}_{k j}^{4 \pi}\right)
$$

The corresponding error term for $\Delta \bar{R}_{i j}^{S}$ is

$$
e\left(\Delta \vec{P}_{i j}^{-s}\right)=\left\{\Sigma_{k=1}^{3}\left[\left(\Delta \bar{R}_{k j}^{4 \pi}\right)^{2} e^{2}\left(s_{i k}\right)+s_{i k}^{2} e^{2}\left(\Delta R_{k j}^{4 \pi}\right)\right]\right\} 1 / 2
$$

where $\Delta \vec{R}_{k j}^{4 \pi}$ and $e^{2}\left(\Delta \vec{R}_{k j}^{4 \pi}\right)$ are defined by equations (5.24) and $(6.25)$, respectively. For $i=4$ there is no Compton stripping; therefore, $\Delta \bar{R}_{4 j}^{S}$ and $e\left(\Delta \bar{R}_{4 j}^{S}\right)$ 
can be taken directly from equations $(6.24)$ and $(6.25) . \quad \vec{R}_{i}$ and $\mu_{j}$ are determined by linearizing equation ( 6.39 ) such that

$$
\ln \left(\Delta R_{i j}^{S}\right)=\ln \left(\bar{R}_{i}\right)+\mu_{j}\left(122-\bar{H}_{j 1}\right)
$$

fixing $j$ and then assigning

$$
\begin{aligned}
x_{j} & =122-\bar{H}_{j 1} \\
e\left(x_{j}\right) & =e\left(\bar{H}_{j 1}\right) \\
Y_{j} & =\ln \left(\Delta \bar{R}_{i j}^{S}\right) \\
e\left[\ln \left(\Delta R_{i j}^{S}\right)\right] & =e\left(\Delta \bar{R}_{i j}^{S}\right) / \Delta \bar{R}_{i j}^{S}
\end{aligned}
$$

and solving the resultant set of equations posed by the MLS regression algorithm given in Appendix $B$.

Output from the MLS algorithm are $\left[\mu_{j}, e\left(\mu_{j}\right)\right]$ and $\left\{\ln \left(\bar{R}_{j}\right), e\left[\ln \left(\bar{R}_{j}\right)\right]\right\}$. Evaluation of past Lake Mead calibration has indicated that, due to inhomogeneities in ground uranium, the coefficient $\mu_{2}$ is somewhat inaccurate. Therefore, $\mu_{2}$ is recalculated by linearly interpolating between $\mu_{1}$ and $\mu_{3}$ based on relative energy differences. The interpolated values for $\mu_{2}$ and its associated uncertainty are calculated from the equations

and

$$
\mu_{2}=\mu_{1}-0.26\left(\mu_{1}-\mu_{3}\right)
$$

$$
e\left(\mu_{2}\right)=\left[0.55 e^{2}\left(\mu_{1}\right)+0.07 e^{2}\left(\mu_{3}\right)\right]^{1 / 2}
$$

The STP 1 inear attenuation coefficients and related uncertainties, as determined from the MLS regression analysis and equations (6.43) and (6.44), are placed in their appropriate positions in the reduction and error propagation equations.

Lake Mead 122-meter system sensitivities for the three radioelements are computed from the equation

$$
k_{i}^{L M}=\bar{R}_{i} / C_{i}^{L M}
$$


with $C_{\dot{j}}^{L M}$ being the assigned Lake Mead KUT ground concentrations given in Table 4. Values for $\bar{R}_{j}$ in equation (6.45) are computed by exponentiating $\ln \left(\bar{R}_{i}\right)$ returned from the MLS regression analysis of equation (5.42). Uncertainties in $k_{i}^{L M}$ are given by

$$
e\left(k_{i}^{L M}\right)=k_{i}^{L M}\left\{e^{2}\left[\ln \left(\bar{R}_{j}\right)\right]+e^{2}\left(C_{i}^{L M}\right) /\left(C_{i}^{L M}\right)^{2}\right\} 1 / 2
$$

Values for $\left[k_{i}^{L M}, e\left(k_{i}^{L M}\right)\right] i=1,2,3$ as computed from equations (5.45) and (6.46) are appropriately placed in the reduction and error propagation equations.

Table 4. Lake Mead Assigned 122-Meter Effective Ground Concentrations ${ }^{2}$

\begin{tabular}{lcccc}
\hline $\begin{array}{c}\text { Radio- } \\
\text { element }\end{array}$ & $\begin{array}{c}\text { Fractional Weight } \\
\text { Unit }\end{array}$ & $\begin{array}{c}\text { Secular } \\
\text { Equilibrium }\end{array}$ & $\begin{array}{c}\text { Concentration } \\
\text { Uncertainty }\end{array}$ \\
\hline Potassium & percent & N/A & 2.53 & 0.47 \\
Uranium & ppm & yes & 2.64 & 0.32 \\
Thorium & ppm & yes & 11.56 & 1.15 \\
\hline
\end{tabular}

Reference: Geodata International, Inc., 1977.

${ }^{b}$ A comprehensive precision analys is was not performed on all ground samples used to derive 122 -meter effective concentrations. Reported uncertainties were taken from intersample variance calculations. Therefore, these uncertainties are more a measure of the inaccuracy involved in assuming Lake Mead to be a uniform ground source than a measure of sample-average imprecision.

A final quality check is performed by analytically continuing Lake Mead 122 -meter sensitivities to zero altitude. Letting $K_{i}^{W F S}$ be defined as

$$
k_{i}^{W F S}=k_{i}^{L M} \exp \left(122 \mu_{i}\right)
$$

values for $k_{i}^{W}$ ES are compared with $k_{i}^{N / N F}$ given in equation (6.14). Past experience has shown that $k_{i}^{i N S}$ and $k_{i}^{W F}$ deviate by less than 15 percent. Any 
deviation greater than 15 percent is sufficient cause to $\mathrm{flag}$ processing data with coefficients determined in paragraph 6.3 until the discrepancy is corrected.

\subsection{STATISTICAL ADEQUACY MEASURE}

Specification BFEC 1200-C, paragraph 4.3.1, calls for a precision calculation to be performed on all single record reduced and statistical analys is assays. Since precision errors for unit radioelement assays are estimated via the error propagation equation, a signal-to-noise ratio (SNR) is readily computable. Therefore, in fulfillment of this requirement, signalto-noise ratios for each of the three radioelement assays are computed by

$$
\operatorname{SNR}_{i}=\left(\bar{C}_{a}\right)_{i} /(\bar{E})_{i}^{1 / 2}
$$

where $\bar{C}_{a}$ and $\bar{E}$ are the final results of applying the reduction and error propagation equations, respectively. Signal-to-noise ratios are computed for both single record reduced and statistical analys is unit sample reductions.

\subsubsection{Quality Flag Codes}

Specification BFEC 1200-C, paragraph 4.7, requires that data quality flags be set for unit sample terrain clearance and ${ }^{40} \mathrm{~K}, 214_{\mathrm{Bi}}$, and ${ }^{208} \mathrm{Tl}$ assays. This is required for both single record reduced and statistical analys is data. Referring to this quality flag field, quality flags are defined as onecharacter fields defined from left to right across the quality flag field as follows:

1. Altitude Flag Code

2. $40_{K}$ Assay Flag Code

3. 214 Bi Assay Flag Code

4. ${ }^{208}$ Tl Assay Flag Code

Flag codes for altitude are defined as the integers $0,1,2$. These codes shall be assigned the following meanings: 
$0 \Leftrightarrow$ sample altitude $>190$ meters

$1 \Leftrightarrow 160$ meters $\leq$ sample altitude $\leq 190$ meters

$2 \Leftrightarrow$ sample altitude $<160$ meters

Altitude is defined as the STP altitude defined in equation (6.26).

Quality flag codes for ${ }^{40} \mathrm{~K}, 214 \mathrm{Bi}$, and ${ }^{208} \mathrm{Tl}$ analys is are

defined as the integers 0 through 9. Integral portions of signal-to-noise ratios as defined in equation (6.48) are computed for each $40_{\mathrm{K}},{ }^{214} \mathrm{Bi}$, and ${ }^{208} \mathrm{Tl}$ single record reduced and statistical analysis assay. Integral parts of SNR are utilized as the quality flag code for the assay, and appropriately placed in the quality flag field. In the event that an integral portion of any assay SNR is greater than nine (9), a value of nine (9) is assigned. The convention adopted for ${ }^{40} \mathrm{~K}, 214_{\mathrm{Bi}}$, and ${ }^{208} \mathrm{Tl}$ assay flags is readily interpretable via standard statistical theory. For example, an assay flag of zero (0) indicates a statistically "inadequate" assay, and a flag of three (3) and above indicates an "adequate" assay. Specific definitions for "adequacy" and "inadequacy" are left to the interpreter, since what is adequate for one interpretation may not be adequate for another. 


\subsection{REFERENCES}

Deming, W. E., 1943, Statistical adjustment of data: New York, John Wiley. Geodata International, Inc., 1977, Lake Mead Dynamic Test Range for calibration of airborne gamma radiation measuring systems: U.S. Department of Energy Open-File Report GJBX-46(77), 2 v.

Ward, D. L., 1978, Construction of calibration pads facility, Walker Field, Grand Junction, Colorado: U.S. Department of Energy Open-File Report GJBX-37(78), $39 \mathrm{p}$.

York, Derek, 1966, Least-squares fitting of a straight line: Canadian Journal of Physics, v. 44, pp. 1079-1086. 


\section{Appendix A}

\section{FITTING A GAUSSIAN SHAPE TO RECOGNIZABLE}

SPECTROMETER PHOTOPEAKS

Averaged $4 \pi$ or $2 \pi$ spectrometer counts for the $k^{\text {th }}$ channel are computed by

$$
\bar{I}_{k}=M^{-1} \Sigma_{i=1}^{M} I_{i k}
$$

where $M$ is the number of unit samples as defined by paragraph 6.3.2. Letting the inclusive channels covering a photopeak and its background be designated by channel numbers $K 1$ and $K 2$, then for $K 1 \leq k \leq K 2$ the curve representing averaged channel intensities is assumed the functional form $f_{k}$ with

$$
f_{k}=a+b k+A \exp \left[-\left(C H^{c}-k\right)^{2} /\left(2 s^{2}\right)\right]
$$

where

$$
\begin{aligned}
a+b k & =1 \text { inear background of photopeak } \\
A & =\text { photopeak amplitude } \\
\mathrm{CH}^{C} & =\text { photopeak centroid channel } \\
S & =\text { photopeak spread }
\end{aligned}
$$

The best estimate for $\left(a, b, A, C H^{c}, s\right)$ which matches $f_{k}$ to $\vec{I}_{k}$ is found by using the least-squares matching criteria and Newton's method of iteration.

Moving to matrix notation, let

$$
\bar{X}=\left(a, b, A, C H^{c}, s\right)^{\top}
$$

An initial guess to $\bar{x}$, called $\bar{x}^{(0)}$, is first computed by

$$
\begin{aligned}
b & =\left(\bar{I}_{K 2}-\bar{I}_{K 1}\right) /(K 2-K 1) \\
a & =\bar{I}_{K 1}-b K 1 \\
C H^{C} & =K C \text { (chosen visually) } \\
A & =\bar{I}_{K C}-(a+b K C) \\
s & =1.0
\end{aligned}
$$


Correction vectors, $\Delta \bar{x}^{(i)}$, are iteratively added to the current solution, $\bar{x}^{(i-1)}$, unt $i 1 \Delta \bar{x}^{(i)}$ approaches zero. In equation form

$$
\bar{x}^{(i)}=\bar{x}^{(i-1)}+\Delta \bar{x}(i)
$$

Iterations are terminated at the conditions

$$
\left|\Delta \bar{x}^{(i)}\right| \leq 0.0001|\bar{x}(i-1)|
$$

The correction vector $\Delta \bar{x}^{(i)}$ is computed by

$$
\Delta \bar{X}^{(i)}=\left.\left(D^{\top} D\right)^{-1} D^{\top} \Delta \bar{Y}\right|_{\bar{X}}(i-1)
$$

$D$ is a $N \times 5$ matrix $(N=K 2-K 1+1)$ whose $k j^{\text {th }}$ el ement is

$$
(D)_{k j}=\partial f_{k} / \partial(\bar{x})_{j}
$$

$\Delta \bar{Y}$ is a $N \times 1$ vector whose $k^{\text {th }}$ el ement is

$$
(\Delta \bar{Y})_{k}=\bar{I}_{k}-f_{k}
$$

Upon converging to a solution $\bar{x}^{(f)}$, errors are estimated by use of the error propagation formula

$$
e^{2}\left\{\left[\bar{X}^{(f)}\right]_{j}\right\}=\Sigma_{k=K I}^{K 2}\left\{\partial\left[\bar{X}^{(f)}\right]_{j} / \partial \bar{I}_{k}\right\} e^{2}\left(\bar{I}_{k}\right)
$$

This equation allows errors to propagate into $\bar{x}^{(f)}$ through precision errors present in averaged channel intensities. Partial derivatives in equation (A.10) are computed in the following manner. Let $\phi_{j}$ functionally represent the $i^{\text {th }}$ el ement of $\left(D^{\top} D\right)^{-1} D^{\top} \Delta \bar{Y}$, or

$$
\phi_{i}=\left[\left(D^{\top} D\right)^{-1} D^{\top} \Delta \bar{Y}\right]_{i}
$$

By the chain rule,

$$
\partial \phi_{i} / \partial \bar{I}_{k}=\Sigma_{j=1}^{5}\left[\partial \phi_{i} / \partial(\bar{X})_{j}\right]\left[\partial(\bar{X})_{j} / \partial \bar{I}_{k}\right]
$$


Since for each $k(K 1 \leq k \leq K 2)$ there are five equations, $\partial \phi_{j} / \partial \vec{I}_{k}$, and five unknowns $a(\bar{X})_{j} / \partial \bar{I}_{k}$, and since $\partial \phi_{j} / \partial \bar{I}_{k}$ and $\partial \phi_{j} / \partial(\bar{X})_{j}$ can be numerically evaluated at the final solution $\bar{x}(f)^{k}, \partial(\bar{x})_{j} / \partial \bar{I}_{k}$ evaluated at the final solution also can be solved for by matrix inversion. This process is repeated $N$ times (i.e., for $k 1 \leq k \leq k 2$ ) for evaluating $\partial\left[\bar{x}^{(f)}\right]_{j} / \partial \bar{I}_{k}$ in equation (A.10). Error in average channel counts in equation (A.10) is estimated by

$$
e^{2}\left(\bar{I}_{k}\right)=\bar{I}_{k} / 11
$$

This equation assumes variations in channel counts obey Poisson counting statistics. 
Appendix B

MULTIPLE LEAST SQUARES REGRESSION ANALYSIS

Given a set of observations and associated uncertainties

$$
\begin{aligned}
Y_{i}, e\left(Y_{i}\right)= & \text { dependent observations and associated errors } \\
& (i=1, M) \\
X_{i j}, e\left(X_{i j}\right)= & \text { independent observations and associated } \\
& \text { errors }(j=1, N)
\end{aligned}
$$

the objective function

$$
y_{i}=\Sigma_{j=i}^{N} a_{j} x_{i j}^{\prime}+b
$$

for unknowns $a_{j}$ and $b$ that best fits $\gamma_{i}$ is customarily solved for by minimizing the least-square distance function $\psi$ where

$$
\psi=\Sigma_{i=1}^{M}\left[w_{i}\left(y_{i}-Y_{j}\right)^{2}\right]
$$

The weighting factors $w_{i}$ that are usually employed are the inverse sum of the square errors in $X_{i j}$ and $Y_{i}$; i.e.,

$$
w_{i}=\left[e^{2}\left(Y_{i}\right)+\Sigma_{j=1}^{N} e^{2}\left(x_{i j}\right)\right]^{-1}
$$

The conventional treatment of this problem assumes that independent variables $x_{i j}$ are known to a greater degree of precision than dependent observations. This is to say

$$
e\left(X_{i j}\right) / X_{i j}<e\left(Y_{i}\right) / Y_{i}
$$

For the one-dimensional case, Deming (1943) proposed that when

$$
e\left(X_{i}\right) / x_{i}=e\left(Y_{i}\right) / Y_{i}
$$

the "best" straight line is given by minimizing

$$
\psi=\Sigma_{i=1}^{n} w\left(X_{i}\right)\left(x_{i}-X_{j}\right)^{2}+w\left(Y_{i}\right)\left(y_{i}-Y_{i}\right)^{2}
$$


subject to the constraint

$$
y_{i}=a x_{i}+b
$$

York (1966) has given an exact solution to Deming's proposed "best" straight line.

The multiple least squares (MLS) algorithm described here is simply a generalization of Deming's constraint and matching criteria to include linear functions of $N$ independent variables.

ceneralizing Deming's ideas, we wish to minimize the function w with

$$
\psi=\Sigma_{i=1}^{i Y}\left[w\left(Y_{i}\right)\left(y_{i}-Y_{j}\right)^{2}+\Sigma_{j=1}^{N} w\left(X_{i j}\right)\left(x_{i j}-X_{i j}\right)^{2}\right]
$$

subject to the constraint

$$
y_{i}=\Sigma_{j=1}^{N} a_{j} x_{i j}+b
$$

As weighting factors, we use

$$
\begin{aligned}
& w\left(x_{i j}\right)=e^{-2}\left(X_{i j}\right) \\
& w\left(Y_{j}\right)=e^{-2}\left(Y_{j}\right)
\end{aligned}
$$

By introducing Lagrange Multipliers $\lambda_{j}$, it can be shown that minimizing equation (B.8) subject to equation (B.9) is equivalent to minimizing the function $\psi^{\prime}$ where

$$
\begin{aligned}
\Psi^{\prime}= & \Sigma_{j=1}^{M}\left\{w\left(Y_{i}\right)\left(y_{i}-Y_{i}\right)^{2}+\Sigma_{j=1}^{i} w\left(X_{i j}\right)\left(x_{i j}-X_{i j}\right)^{2}\right. \\
& \left.+\lambda_{i}\left[y_{i}-\left(\Sigma_{j=1}^{N} a_{j} x_{i j}+b\right)\right]\right\}
\end{aligned}
$$

Taking the partial derivatives of $\psi^{\prime}$ with respect to all unknowns $\left(a_{j}, b, x_{i j}, y_{i}, \lambda_{i}\right)$, setting each to zero, and then substituting where possibie leads to $i+1$ nonitinear equations and $i+1$ unknowns. These nonlinear equations are for $j=1, \%$

$$
\Sigma_{i=1}^{M} \lambda_{i} x_{i j}+\lambda_{i}^{2} a_{j} /\left[2 w\left(x_{i j}\right)\right]=0
$$


and

$$
\Sigma_{i=1}^{M} \lambda_{i}=0
$$

The expression for $\lambda_{i}$ is

$$
\lambda_{i}=u_{i} / v_{i}
$$

with

$$
\begin{gathered}
u_{i}=Y_{i}-\Sigma_{j=1}^{N} a_{j} X_{i j}-b \\
v_{i}=\left[2 w\left(Y_{j}\right)\right]^{-1}+\sum_{j=1}^{N} a_{j}^{2} /\left[2 w\left(X_{i j}\right)\right]
\end{gathered}
$$

The coefficients $a_{j}$ and b satisfying equations (B.12) and (B.13) are numerically solved for by Newton's method of iteration using the conventionai weighted-least-squares solution as an initial guess.

It can be shown that the best estimates for the values for $Y_{i}$ and $X_{i j}$ consistent with minimizing equation (B.i1) are

$$
y_{i}=Y_{i}-\lambda_{i} /\left[2 w\left(Y_{i}\right)\right]
$$

and

$$
x_{i j}=x_{i j}+\lambda_{i} a_{j} /\left[2 w\left(x_{i j}\right)\right]
$$

Values for $y_{i}$ and $x_{i j}$ can be compared to data values $y_{i}$ and $x_{i j}$ and used as a measure of the deviation of $Y_{i}$ and $X_{i j}$ from the best-fit plane.

Assuming $a_{j}$ and $b$ to be functions of $X_{i j}$ and $Y_{i}$ alone, the formulas for propagating error into $a_{j}$ and $b$ due to errors in $X_{i j}$ and $Y_{i}$ are given by

$$
e^{2}\left(a_{j}\right)=\Sigma_{i=1}^{M}\left[\left(\partial a_{j} / \partial Y_{i}\right)^{2} e^{2}\left(Y_{i}\right)+\Sigma_{k=1}^{N}\left(\partial a_{j} / \partial X_{i k}\right)^{2} e^{2}\left(X_{i k}\right)\right]
$$


and

$$
e^{2}(b)=\Sigma_{i=1}^{M}\left[\left(\partial b / \partial Y_{i}\right)^{2} e^{2}\left(Y_{i}\right)+\Sigma_{k=1}^{N}\left(\partial b / \partial X_{i k}\right)^{2} e^{2}\left(X_{i k}\right)\right]
$$

Partial derivatives in equations (B.18) and (B.19) can be numerically computed in the following way. Let $\phi_{k}$ functionally represent the equations on the left-hand side of equations (B.12) and (B.13), and let $Z$ denote the variable $X_{i j}$ or $Y_{i}$ for some $i j$. By the chain rule,

$$
\partial \phi_{k} / \partial Z=\sum_{j=1}^{N}\left(\partial \phi_{k} / \partial a_{j}\right)\left(\partial a_{j} / \partial Z\right)+\left(\partial \phi_{k} / \partial b\right)(\partial b / \partial Z)
$$

Since there are $N+1$ such linear equations $(k=1, N+1)$ and since $\partial \phi_{k} / \partial Z$, $\partial \phi_{k} / \partial a_{j}$, and $\partial \phi_{k} / \partial b$ can be numerically computed at the solution to equations $(B .12)$ and $(B .13)$, and since there are $N+1$ unknowns $\left(\partial a_{j} / \partial Z\right.$ and $\partial b / \partial Z)$, the unknowns can be solved for by matrix inversion. This allows a numerical evaluation for equations (B.18) and (B.19). 
Appendix C

PREPARATION OF AVERAGE RAW WINDOW COUNT RATES,

RELATED UNCERTAINTIES, AND POISSON COUNT-RATE QUALITY CHECK

Let $x_{j}$ represent the flight-line time series for some $4 \pi$ or $2 \pi$ raw window count rate, or other observed quantity such as temperature, pressure, altitude, or live time. The average value for such a quantity is

$$
\bar{x}=M^{-1} \Sigma_{i=1}^{M} x_{i}
$$

where $M$ is the number of observations in the time series. The sample variance is given by

$$
s^{2}=(M-1)^{-1} \Sigma_{i=1}^{M}\left(x_{i}-\bar{x}\right)^{2}
$$

and the error estimate in $\vec{x}$ is given by

$$
e(\bar{x})=s /(M)^{1 / 2}
$$

If $x_{i}$ represents a raw window count rate and if this count rate shows Poisson statistical fluctuations, then for flight lines with nonvarying sources

$$
\left|s-(\bar{x} / L)^{1 / 2}\right| \leq\left\{s /(2 M)^{1 / 2}+[\bar{x} /(M L)]^{1 / 2}\right\}
$$

where $L$ is the average spectrometer live time for the flight line. This quality check is a measure of the amount of noise introduced by the spectrometer hardware.

If the inequality in equation (C.4) is not met for all average raw window count rates and all flight lines collected at high altitude or the Walker Field pads, this is sufficient cause to disqualify the subcontractor's system from participating in NURE aerial surveys. 